\author{
Military Technical College \\ Kobry El-Kobbah, \\ Cairo, Egypt
}

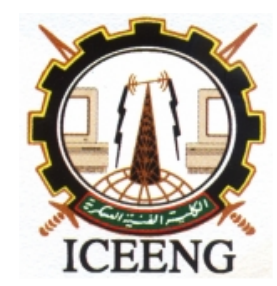

\author{
$8^{\text {th }}$ International Conference \\ on Electrical Engineering \\ ICEENG 2012
}

\title{
A Newly Proposed Routing Protocol for Wireless Mesh Networks (WMNs)
}

\author{
By \\ Marw.M.Khashaba* \\ Prof.Dr.Fathy.A.Amer** \\ Dr.Nabil.A.Lashin $* * *$
}

\section{$\underline{\text { Abstract: }}$}

WMNs are undergoing rapid commercialization in many application scenarios such as providing internet access, extending the coverage of wireless local area networks (WLANs), community networking, building automation, high speed metropolitan area networks, and enterprise networking. WMNs are one of the few commonly implemented types of mobile ad-hoc networks (MANETs), so general MANET routing protocols can be used in WMNs. While general MANET routing protocols can be used in WMNs, it is expected that a protocol that takes the particularities of WMNs into account will outperform the general protocol. This paper surveys recent routing protocols for MANET routing protocols and discuss the new WMNs routing protocols recent used in market. A new routing protocol is proposed uses all WMNs consideration into account. The proposed routing protocol is based on source routing mechanism, showing that by preferring stable routes, the backbone of stationary nodes presented in WMNs can be effectively used to improve the performance of routing protocol, so the proposed protocol will be called Source Route Routing Protocol (SRRP). The main goal of the proposed protocol is to improve throughput and to reduce average end-to-end delay and packet delivery loosing ratio in large networks. The mobility of the nodes will be maintained having a significant improvement in different network sizes than in MANET routing protocols.

\section{Keywords:}

Wireless Mesh Networks (WMNs); mobile ad-hoc networks (MANETs); Routing 


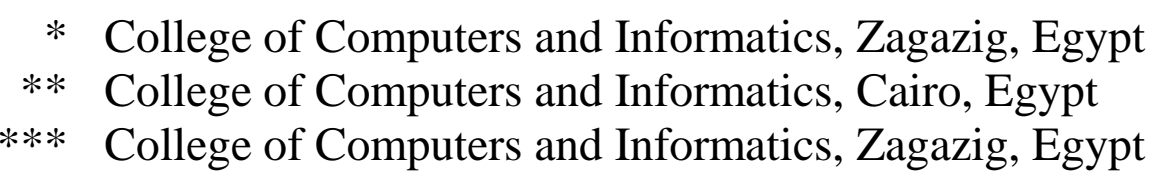

\section{Introduction:}

In recent years, the number of wireless-enabled devices such as laptops, PDAs, and mobile phones has been growing rapidly. At the same time, the Internet has turned into a critical networking infrastructure and continuous access is highly demanded from end users. Combined, these developments constitute a strong demand for Internet access from mobile devices anywhere, anytime. With current wireless technology, the gap between a set of wireless devices and the Internet is bridged by fixed wireless stations, so-called access points. Such access points establish a network among the wireless devices and bridge this network with the Internet. However, the communication range of current commodity wireless LAN technology is limited to a few hundred meters and complete coverage of a city would require a prohibitively high number of access points. A solution to extend the coverage of the existing access points is to let wireless devices relay packets towards access points on behalf of their neighbors. Such multi-hop wireless access networks are called wireless mesh networks (WMN) in the remainder of this paper.

Wireless Mesh Networks (WMNs) [1]-[3] are a relatively new wireless multihop technology that has much in common with the mobile ad hoc networks (MANETs). In its general form, a WMN is a set of wireless nodes that can communicate with each other, forwarding each other's packets. Like in MANETs, each node is both a host and a wireless router. Clients can connect to the WMN routers using common networking interfaces (e.g., Ethernet, 802.11, Bluetooth) or, in some cases, a PCI or a PCMCIA bus (i.e., the WMN router is embedded in a network card). Routing is a fundamental characteristic of WMNs. The routing protocol's strengths and weaknesses are reflected directly in the WMN's characteristics. So routing protocol that made especially for WMN taking into account all its characteristics and topology changes can reach all WMNs expectations. The primary goal of a routing protocol is correct and efficient route establishment between a pair of nodes so that messages may be delivered in a timely manner. Route construction should be done with a minimum of overhead and bandwidth consumption. A Mobile Ad-hoc Network (MANET) is a temporary wireless network composed of mobile nodes, in which an infrastructure is absent. There are no dedicated routers, servers, access points and cables. In this paper, we introduce SRRP, a source route routing protocols especially designed for WMNs, based on source routing mechanism, showing that by preferring stable routes, the backbone of stationary nodes presented in WMNs can be effectively used to improve the performance of routing 
protocol. The paper is organized as follow. In section 2, we will briefly summarize WMNs definitions, uses, advantages and architecture. In section 3, MANET networks will be discussed and a comparison between it and WMNs will be discussed. In section 4, routing protocols for MANET will be described, discussed, and compared with each other. In section 5, a new routing protocol especially for WMNs is proposed; the proposed protocol theory and algorithm will be described and discussed. In section 6 , a comparison between the proposed routing protocol and two of MANET routing protocol (AODV as an on-demand routing protocol and WRP as a table-driven routing protocol) is performed. The proposed protocol will be evaluated via simulation in terms of WMNs changes, as number of hops, node placements, and mobility existence. Simulation results of the comparison will be discussed. Finally, a conclusion is given in section 6 and a brief future work is given in section 7 .

\section{Wireless Mesh Networks (WMNs):}

Wireless mesh networks (WMNs) have emerged as a key technology for nextgeneration wireless networking. Because of their advantages over other wireless networks, WMNs are undergoing rapid progress and inspiring numerous applications. However, many technical issues still exist in this field. In order to provide a better understanding of the research challenges of WMNs, this chapter presents a detailed investigation of current state-of-the-art protocols and algorithms for WMNs. In the next section(s), a briefly defined of the WMN will be mentioned; its benefits to the community, and its types and applications will be discussed.

Mesh is a network topology where devices are connected via many links and select the optimal path to forward traffic as shown in figure 1 and figure 2. WMNs draw an increasing attention in nowadays research and industry. It is a promising wireless technology for several emerging and commercially interesting application, e.g., broadband home networking, community and neighborhood networks, coordinated network management, intelligent transportation systems. It is gaining significant attention as a possible way for Internet service providers (ISPs) and other end-users to establish robust and reliable wireless broadband service access at a reasonable cost.

Wireless mesh networks (WMNs) are dynamically self-organized and self- configured; with the nodes in the network automatically establishing an ad hoc network and maintaining the mesh connectivity. WMNs are comprised of two types of nodes: mesh routers and mesh clients. Other than the routing capability for gateway/bridge functions as in a conventional wireless router, a mesh router contains additional routing functions to support mesh networking. Through multi-hop communications, the same coverage can be achieved by a mesh router with much lower transmission power. To further improve the flexibility of mesh networking, a mesh router is usually equipped with 
multiple wireless interfaces built on either the same or different wireless access technologies.

A WMN operates like a network of fixed routers, except that routers are connected only by wireless links. A WMN plays mainly the role of a backbone network. It can interconnect or provide access to the Internet or otherwise line backbones to its mesh clients. WMNs are dynamically self-organized and self-configured, with the nodes in the network automatically establishing an ad hoc network and maintaining the mesh connectivity. A WMN is self-forming/self-healing and selects the optimal path back to the "wired" network.

\subsection{Wireless Network versa Wired Network:}

Different from traditional wireless networks, WMN is dynamically self-organized and self-configured. In other words, the nodes in the mesh network automatically establish and maintain network connectivity. This feature brings many advantages for the endusers, such as low up-front cost, easy network maintenance, robustness, and reliable service coverage. In addition, with the use of advanced radio technologies, e.g., multiple radio interfaces and smart antennas, network capacity in WMNs is increased significantly. Moreover, the gateway and bridge functionalities in mesh routers enable the integration of wireless mesh networks with various existing wireless networks, such as wireless sensor network, wireless-fidelity (Wi-Fi), and WiMAX. Consequently, through an integrated wireless mesh network, the end-users can take the advantage of multiple wireless networks. Furthermore the main advantages of WMN s in comparison to traditional broad band Internet access technologies (cable-modem and xDSL) is the dramatically reduced initial metropolitan area network is the market coverage (especially in areas with significant obstructions - trees, high-rise buildings) and reliability (multiple available routes can avoid failed nodes and poor links).

Figure (1): Mesh Topology

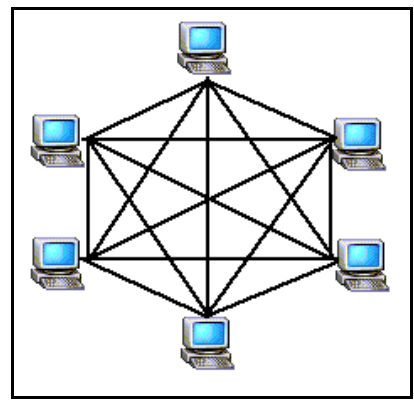


Figure (2): Simple Wireless Mesh Topology

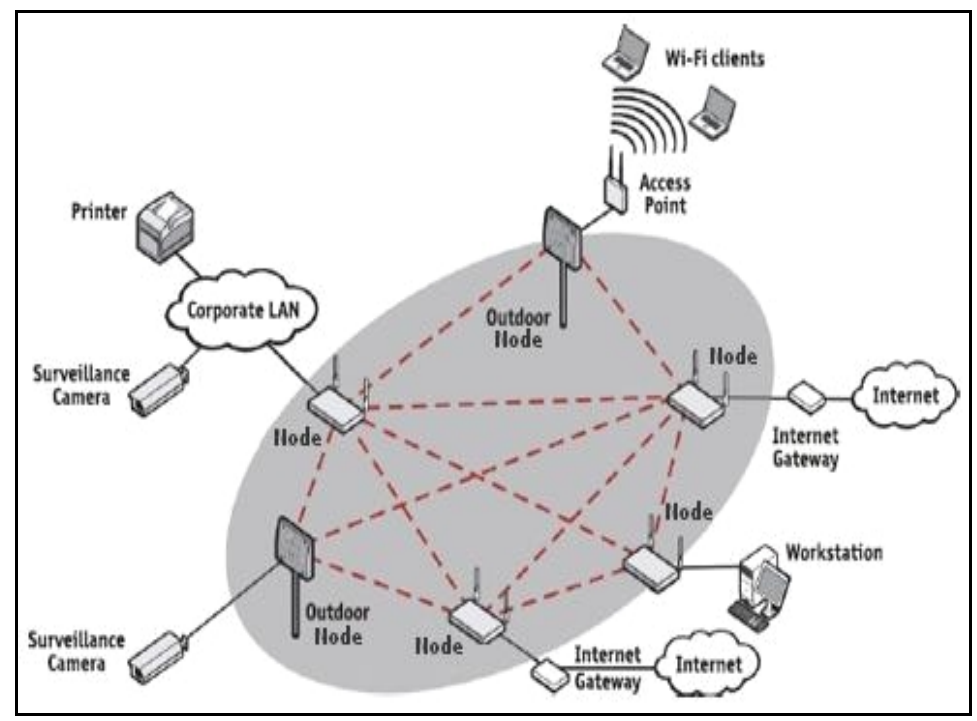

\subsection{Advantages of Wireless Mesh Networks:}

Nowadays the development of the next-generation wireless systems (e.g., the fourth generation (4G) mobile cellular systems, IEEE $802.11 n$, etc.) aims to provide high data rates in excess of $1 \mathrm{Gbps}$. Thanks to its capability of enhancing coverage with low transmission power, wireless mesh networks (WMNs) play a significant role in supporting ubiquitous broadband access [1-8]. Some of the benefits and characteristics of wireless mesh networks are highlighted as follows:

\section{1) Reliability}

In WMNs, the wireless mesh routers provide redundant paths between the sender and the receiver of the wireless connection

2) Low Installation Costs

WMN can be rapidly deployed in a large-scale area with a minimal cabling engineering work so as to lower the infrastructure and deployment costs.

3) Large Coverage Area

Currently, the data rates of wireless local area networks (WLANs) have been increased, e.g., $54 \mathrm{Mbps}$ for $802.11 \mathrm{a}$ and $802.11 \mathrm{~g}$, by utilizing spectrally efficient modulation schemes.

\section{4) Automatic Network Connectivity}

Wireless mesh networks are dynamically self-organized and self-configured. In other words, the mesh clients and routers automatically establish and maintain network connectivity, which enables seamless multi-hop interconnection service [4]. 


\section{5) Robustness}

Due to multiple paths for each node, an appealing feature of WMNs is its robustness [9]. If some nodes fail, the mesh network can continue operating by forwarding data traffic via the alternative nodes.

\section{6) Flexibility}

WMN can concurrently support a variety of wireless radio access technologies, thereby providing the flexibility to integrate different radio access networks [10].

\section{7) Organization and business models}

The decentralized nature of mesh networks lends itself well to a decentralized ownership model wherein each participant in the network owns and maintains their own hardware, which can greatly simplify the financial and community aspects of the system.

\section{8) Integration}

Mesh hardware is typically small, noiseless, and easily encapsulated in weatherproof boxes.

\section{9) Reality fit}

Reality rarely comes as a star, ring, or a straight line. In difficult terrain where not every user can see one or few central points, chances are she can see one or more neighboring users [11].

Therefore, WMNs diversify the capabilities of ad-hoc networks instead of simply being another type of ad hoc network. These additional capabilities necessitate new algorithms and design principles for the realization of WMNs.

\subsection{Network Architecture [12, 13]:}

A typical wireless mesh network consists of mesh routers and mesh clients as shown in figure 3. In this architecture, while static mesh routers form the wireless backbone, mesh clients access the network through mesh routers as well as directly meshing with each other. Unlike a traditional ad hoc network, which is an isolated self configured wireless network, the mesh network architecture introduces a hierarchy with the implementation of dedicated and power enabled mesh routers. In this integrated network architecture, some of the mesh routers are also called as gateways, which are special wireless routers with a high-bandwidth wired connection to the Internet.

More specifically, mesh routers contain advanced routing functionalities to support mesh networking. This feature of mesh routers is realistic, since mesh routers are fixed nodes, with no constraints on power supply (since they are assumed to be connected to power lines), with multiple wireless interfaces built on either the same or different wireless access technologies.

Different from mesh routers, mesh clients can be mobile nodes, which typically run 
on batteries. Thus, power usage of mesh clients should be limited. This can be achieved by means of reduced radio functions, e.g., single wireless interface, low antenna gain, and low computational complexity. The target technology for both mesh routers and mesh clients is the IEEE 802.11, which is a well known standard for Wireless Local Area Networks WLANs. The main reason is the widespread availability of 802.11 devices, which allows a fast deployment of WMNs by using off the shelf solutions5. However, to leverage on this opportunity, the modifications required by mesh routers and mesh clients should be aware of the existing hardware constraints and limitations. Mesh routers are resource rich nodes equipped with high processing and memory capabilities, while mesh clients have limited memory and computational power.

The architecture of WMNs introduces a hierarchy with the implementation of the dedicated and power enable mesh routers; it can be classified into three types see figure 3:

a) Infrastructure/Backbone WMNs.

b) Client WMNs

c) Hybrid WMNs

Figure (3): WMN Architecture

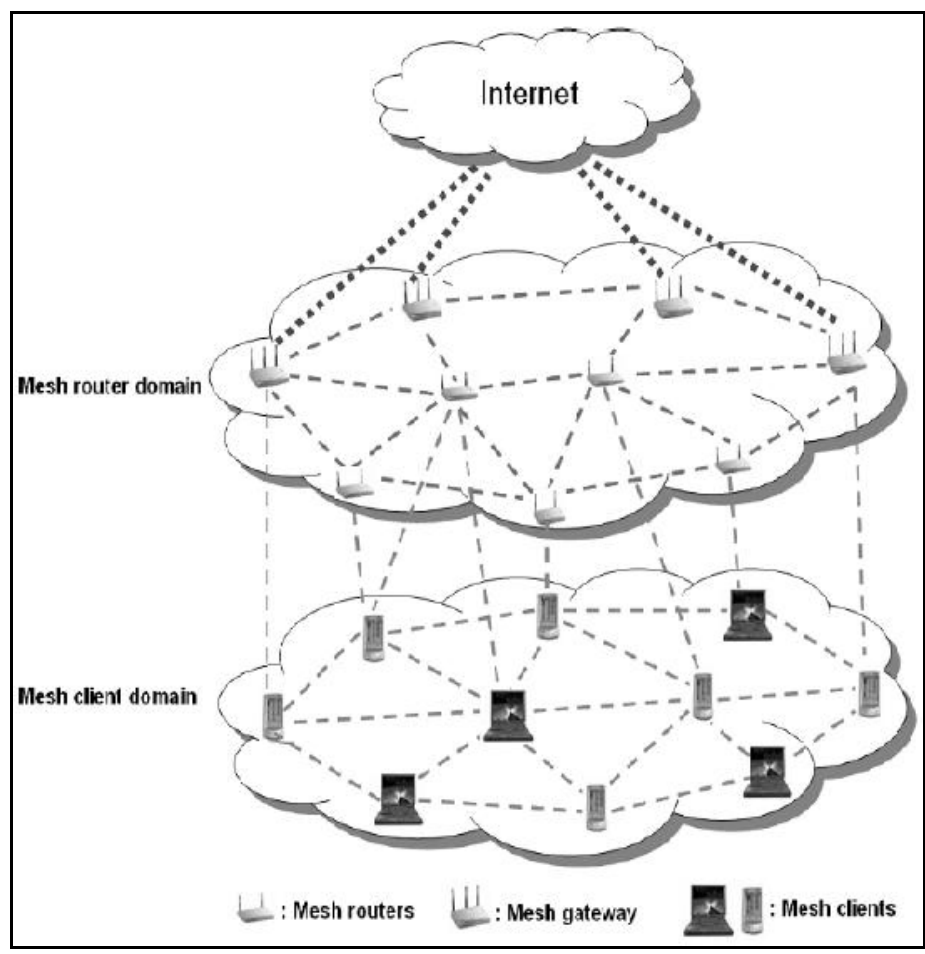




\section{Mobile Ad-Hoc Networks (MANET):}

An ad hoc network is a group of wireless mobile devices (nodes) that communicate with each other in a collaborative way, over multi-hop wireless links, without any stationary infrastructure or centralized management e.g., no base station see figure 4. Examples of ad hoc networks include: disaster situations such as earthquake and flooding, where the rescue teams need to coordinate themselves without the availability of fixed networks; soldiers in a battlefield exchanging tactical information; entrepreneurs in a meeting sharing business information [14]. A mobile ad-hoc network (MANET) is a selfconfiguring infrastructure-less network of mobile devices connected by wireless links. The high mobility and low bandwidth features of MANET networks make it necessary for a routing protocol to be dynamic and bandwidth efficient to enable the delivery of data packets while producing low control overhead.

Figure (4): Mobile Ad-Hoc Network MANET

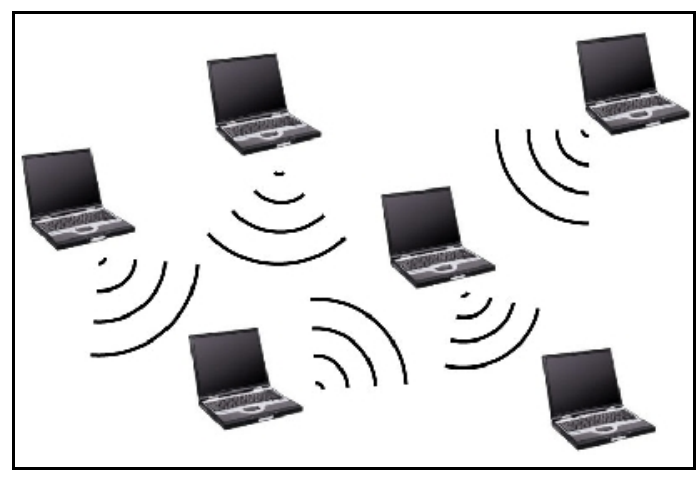

\subsection{Differences between WMNs and General MANETs}

Selecting the "best" routes for different traffic classes is an essential ingredient for QoS support. Taxonomically, WMNs are a particular type of mobile ad hoc network (MANET) [14], [15]. WMNs share the same multihop characteristics and mobilityrelated issues as MANETs.

However, there are also significant differences between WMNs and general MANETs.

\section{1) Gateways}

Most WMNs are designed to provide connectivity to a distribution system (usually connected to the Internet). Therefore, they have specialized nodes (the gateways) that provide connectivity to the distribution system. 


\section{2) Traffic pattern}

In WMNs, most of the traffic is expected to flow between the clients and the Internet (via the gateways). In general MANETs, the common assumption is that any node is equally likely to be the source or the destination of a traffic flow. In another word, in WMNs, most of the traffic originates or terminates at the gateways (nodes connected to the wired infrastructure/Internet).

\section{1) Mobility}

In most WMNs, nodes belong to two distinct categories: either stationary (e.g., on lamp poles, rooftops, etc.) or mobile, capable of roaming in the coverage area provided by the stationary nodes. In MANETs, it is often assumed that all nodes have homogeneous mobility characteristics.

Consequently, instead of being another type of ad-hoc networking, WMNs diversify the capabilities of ad-hoc networks. Therefore the characteristics and requirements of WMNs are considerably different than those of general MANETs such that a new routing protocol can significantly outperform the general MANET routing protocols

\subsection{Why WMNs use Protocols designed for MANET networks?:}

Also there are a lot of differences between WMNs and MANET; MANET protocols consider the best choice for WMN. WMN and MANET have some similarity:

1) The backbone for WMNs is similar to that in the MANET, hence; each user client node operates not only as a host but also as a router.

2) Use's packets are forwarded to and from internet connected gateway in multihop fashion

\section{Routing Protocol:}

Packets are to be routed from the source to destination may traverse through many cross points called routers. A router is a specialized computer that is equipped with hardware/ software for packet processing. It is also equipped for processing of routing protocols and can handle configuration requirements. A router is architected differently depending on its role in a network, such as a core router or an edge router, although all routers have a common set of requirements [16].

There are a large number of protocols and implementations in the field of mesh and adhoc networking, each with differing goals and design criteria, and more are being developed every day. It is beyond the scope of this chapter to give a comprehensive list of available protocols and systems. Many of existing routing protocols have been standardized by IETF and have been in use for many years. Some of those protocols have proven themselves in the Internet and are expected to continue to perform well for many years to come. 


\subsection{Routing Protocols Types:}

The primary goal of a routing protocol is correct and efficient route establishment between a pair of nodes so that messages may be delivered in a timely manner. Route construction should be done with a minimum of overhead and bandwidth consumption.

Routing protocols for Mobile ad hoc networks can be classified into three main categories [17]:

1) Proactive or table-driven routing protocols.

2) Reactive or on-demand routing protocols.

3) Hybrid routing protocol.

\section{1) Table-Driven Routing Protocols}

Table-driven routing protocols attempt to maintain consistent, up-to-date routing information from each node to every other node in the network. The routing information is kept in a number of different tables and they respond to changes in network topology by propagating updates throughout the network in order to maintain a consistent network view. The areas in which these protocols differ are the way the routing information is updated, detected and the type of information kept at each routing table. The table-driven ad hoc routing approach is similar to the connectionless approach of forwarding packets, with no regard to when and how frequently such routes are desired. It relies on an underlying routing table update mechanism that involves the constant propagation of routing information. Moreover proactive protocols find route between all source destination pairs regardless of the use or need for such routes. DSDV [18], STAR [19] and WRP [20] are examples of table-driven routing protocol.

2) On-Demand Routing Protocols

On-demand routing protocols were designed to reduce the overheads in Table-Driven protocols by maintaining information for active routes only. When a node requires a route to a destination, it initiates a route discovery process within the network. This process is completed once a route is found or all possible route permutations have been examined.

Once a route has been established, it is maintained by a route maintenance procedure until either the destination becomes inaccessible along every path from the source or until the route is no longer desired. Route discovery usually occurs by flooding a route request packets through the network. When a node with a route to the destination (or the destination itself) is reached a route reply is sent back to the source node using link reversal if the route request has traveled through bi-directional links or by piggybacking the route in a route reply packet via flooding. When a node using an on-demand protocol desires a route to a new destination, it will have to wait until such a route can 
be discovered. Moreover reactive protocols initiate routing activities only in the presence of data packet in need of a route. AODV [21] and DSR [22] are on-demand protocol examples. A simple comparison between on-demand and table-driven routing protocols is presented in table1.

3) Hybrid Routing Protocol [23]

Hybrid protocols seek to combine the proactive and reactive approaches. An example of such a protocol is the Zone Routing Protocol (ZRP). ZRP divides the topology into zones and seek to utilize different routing protocols within and between the zones based on the weaknesses and strengths of these protocols.

Table (1): Comparison of Table-Driven and On-Demand Routing Protocols.

\begin{tabular}{|l|l|l|}
\hline Parameter & Table-Driven & On-Demand \\
\hline Route availability & Always available & Computed when needed \\
\hline Periodic updates & Always required & Not required \\
\hline $\begin{array}{l}\text { Control traffic } \\
\text { generated }\end{array}$ & $\begin{array}{l}\text { Usually higher than On- } \\
\text { demand }\end{array}$ & $\begin{array}{l}\text { Increases with mobility of active } \\
\text { routes }\end{array}$ \\
\hline $\begin{array}{l}\text { Storage } \\
\text { requirements }\end{array}$ & Higher than On-demand & $\begin{array}{l}\text { Depends on the number of routes } \\
\text { maintained or needed }\end{array}$ \\
\hline Delay & $\begin{array}{l}\text { Small as routes are } \\
\text { predetermined }\end{array}$ & $\begin{array}{l}\text { High as routes are computed when } \\
\text { needed }\end{array}$ \\
\hline Scalability & Usually up to 100 nodes & Usually higher than Table-Driven \\
\hline
\end{tabular}

\subsection{Routing Protocol Examples:}

\section{A) On-Demand Routing Protocol}

\section{1) Ad Hoc On-Demand Distance Vector Routing (AODV)}

The Ad Hoc On-Demand Distance Vector routing protocol (AODV) is an improvement of the Destination-Sequenced Distance Vector routing protocol (DSDV). It is based on distance vector and also uses the destination sequence numbers to determine the freshness of the routes. It operates on the On-demand fashion. AODV requires hosts to maintain only active routes. The advantage of AODV is that it tries to minimize the number of required broadcasts. It creates the routes on an on-demand basis, as opposed to maintain a complete list of routes for each destination. Therefore, the literature on AODV, classifies it as a pure on-demand route acquisition system. The 
usage of the AODV protocol for mobile ad hoc networking applications provided consistent results for large scale scenarios.

\section{B) Table-Driven Routing Protocol}

\section{1) Wireless routing Protocol (WRP)}

Wireless Routing Protocol (WRP) is a pro-active protocol that maintains routing information through the exchange of triggered and periodic updates. A node that successfully receives an update message transmits an acknowledgement back to the sender, indicating the link is still viable. In the event that a node has not transmitted anything within a specified period of time, it must transmit a Hello message (instead of exchanging the entire route table) to ensure connectivity. Otherwise, the lack of messages from a node indicates the failure of that link. When a node receives a Hello message from a new node, it sends that neighbor a copy of its routing table information. Each node maintains a distance table, a routing table, a link-cost table, a message retransmission list. The implementation of WRP in GloMoSim (see chapter four) is based upon the pseudo code.

However, the relatively large number of tables that this protocol requires imposes tight memory constraints on the mobile devices, especially when the network begins to grow. This in turn places greater demands on the battery power of the mobile device, and in the case of the GloMoSim, it happens some-times that simulations of more than 100 nodes result in a segmentation core error. In such cases, parameter NUMBER-OFNODES must be set to 99 .

\subsection{Current Used Routing Protocols for WMNs:}

According to the great interest of WMN in the market nowadays, new routing protocols made especially for it for example ROMER routing protocol, AODV-ST, and Orthogonal Rendezvous Routing Protocol.

\section{1) ROMER (Resilient Opportunistic Mesh Routing)}

ROMER [24] is a credit-based, opportunistic forwarding mechanism capable of using multiple routes at short time-scales and building long-term reliable routes at a cost of some redundancy. It is shown that for WMNs with significant variability in link quality, ROMER can significantly improve the packet delivery ratio of the network.

\section{2) AODV-ST}

AODV-ST [25] is a hybrid routing protocol designed for WMNs. It uses proactive tree formations by using a beaconing protocol initiated at the gateways to form spanning trees rooted at the gateway. For intra-mesh routing, it uses AODV to discover routes 
that bypass the gateways. Similar to our work, the main assumption is that the commoncase traffic is to and from the gateway, and, thus, uses the proactively constructed trees. In contrast to our work, the common-case protocol (that forms the routing trees) is proactive, periodically flooding the network (AODV, used for intra-mesh routing, also floods RREQ messages).

\section{3) ORRP (Orthogonal Rendezvous Routing Protocol)}

ORRP [27] assumes that each node has directional communication capability and can therefore have a local sense of direction (i.e. orientation of neighbors is known based on a local North). Notice that this is an even weaker form of information than a global sense of direction (i.e. orientation of neighbors is known based on a global North) which necessitates additional hardware such as a compass.

\section{Proposed Routing Protocol for WMNs:}

The introduced routing protocol follows the simple notion of on-demand routing and uses explicit "source routing" which is why the proposed routing protocol will be called "SRRP" (Source Route Routing Protocol).

\subsection{The Proposed Routing Protocol Theory:}

The proposed protocol (SRRP) uses explicit "source routing", in which a node $S$ wishing to send a packet to a destination node D, has to obtain a source route to D. When some source node originates a new packet addressed to some destination node, the source node places in the header of the packet a "source route" giving the sequence of hops that the packet is to follow on its way to the destination. The sender will obtain a suitable source route by searching its "Route Cache" of routes previously learned; if no route is found in its cache, it will initiate the Route Discovery protocol to dynamically find a new route to this destination node.

\subsection{SRRP Algorithm:}

Suppose a node A is attempting to discover a route to node E. The Route Discovery initiated by node $\mathrm{A}$ in this example would proceed as follows:

A) To initiate the Route Discovery, node A transmits a "Route Request" as a single local broadcast packet, which is received by (approximately) all nodes currently within wireless transmission range of $\mathrm{A}$, including node $\mathrm{B}$ and $\mathrm{C}$ in this example see figure 5 .

Each Route Request identifies the following steps:

1. The initiator of the Route Discovery. 
2. The target of the Route Discovery.

3. A unique request identification (or sequence number), determined by the initiator of the Request.

4. The hop count.

A record listing the address of each intermediate node through which this particular copy of the Route Request has been forwarded. This route record is initialized to an empty list by the initiator of the Route Discovery. In this example, the route record initially lists only node A.

Figure (5): Proposed Network

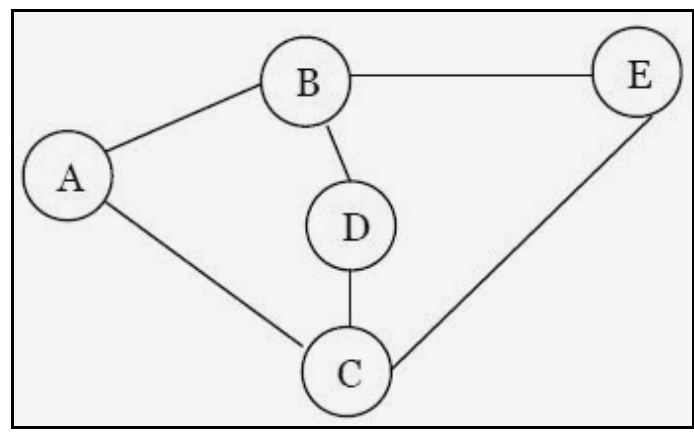

B) The recipient of this Route Request (such as node B in this example), can either be:

1) The target of the Route Discovery, in which case it returns a "Route Reply" to the initiator of the Route Discovery, giving a copy of the accumulated route record from the Route Request. When the initiator receives this Route Reply, it caches this route in its Route Cache for use in sending subsequent packets to this destination.

2) Not the target. In which case:

a) The node checks to see: 1) If it has recently seen another Route Request message from this initiator bearing this same request identification and target address (Note: due to flooding, D receives the same request from both $\mathrm{B}$ and $\mathrm{C}$ ), then the node discards the Request. Or 2) if this node's own address is already listed in the route record in the Route Request; the node also discards the Request.

b) Otherwise, this node appends its own address to the route record in the Route Request (Which is now A, B) and propagates it by transmitting it as a local broadcast packet (with the same request identification). In this example, node B broadcasts the Route Request, and the receiving nodes, in turn, broadcast the Request, resulting in receipt of a copy of the Request by node E. The route record 
is $\mathrm{A}, \mathrm{B}, \mathrm{E}$.

In returning the Route Reply to the initiator of the Route Discovery, Node E could simply reverse the sequence of hops in the route record that it is trying to send in the Route Reply and use this as the source route on the packet carrying the Route Reply itself, as shown in figure 6. Hence, a Route Reply contains:

a) Source Address of the Route Reply. (i.e. Destination of the route requested),

b) The target of the Route Reply (i.e. Source of the route requested).

c) The hop count.

d) The record listing the path from the source of the route to the destination.

When initiating a Route Discovery, the sending node saves a copy of the original packet (that triggered the discovery) in a local buffer. This Buffer contains a copy of each packet that cannot be transmitted by this node because it does not yet have a source route to the packet's destination. Each packet in the Buffer is logically associated with the time that it was placed into it, and is discarded after residing in the Buffer for some timeout period.

The RREP message contains the route so, the data can be transmitted. A route failure may occurred, in that case a route maintenance function will be called sending a route error message (RERR) back to the source as shown in figure 7 . With the information in the RREP message the source node can select alternative routes that don't include the failure node.

Hence, SRRP has three types of packets; Route Request (RREQ), Route Reply (RREP) and Route Error (RERR), see figure 8 and figure 9. The fields of each packet are as described above. Also, the following values are assumed:

- Maximum length of the source route is 9, 7,5 and 3 (hops).

- Broadcast jitter to avoid collisions is $10 \mathrm{msec}$ as default.

- Maximum time for buffering a packet is (11110sec).

- Maximum TTL is 255 (hops).

- A Route Request seen entry is kept for a maximum of $30 \mathrm{sec}$.

Figure (6): RREQ and RREP Message

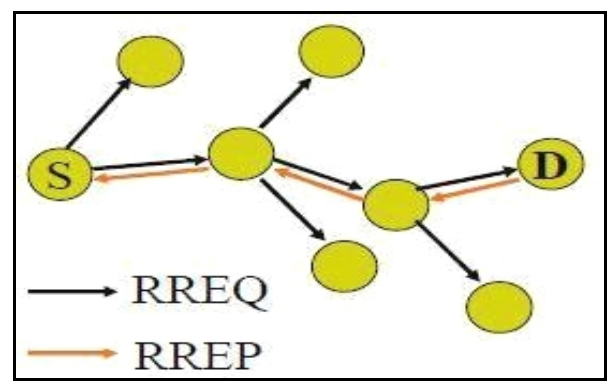


Figure (7): Data and RERR Message

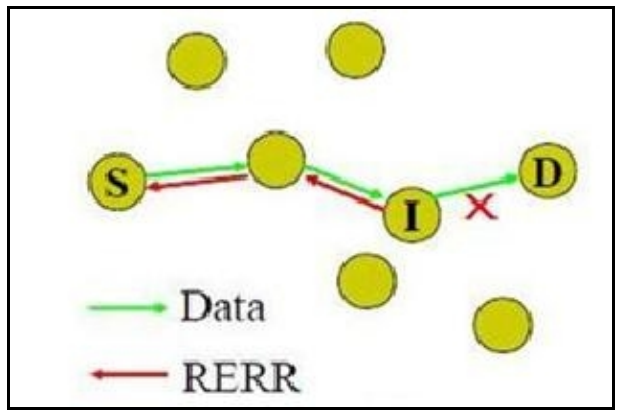

Figure (8): Route Request RREQ Packet

\begin{tabular}{|c|c|c|}
\hline type & ctrl $\quad$ reserved & hopcount \\
\hline \multicolumn{2}{|c|}{ destination IP } \\
\hline \multicolumn{2}{|c|}{ destination sequence number } \\
\hline \multicolumn{2}{|c|}{ source IP } \\
\hline source sequence number
\end{tabular}

Figure (9): Route Reply RREP

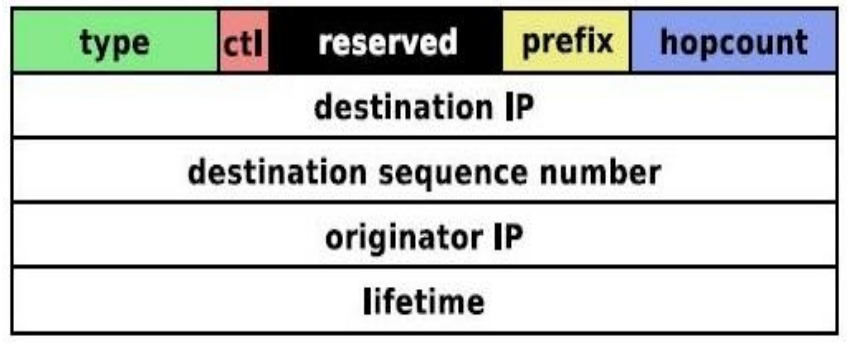

\section{Simulation and Performance Evaluation:}

In order to compare between on-demand and table-driven routing protocols, Mobile Information Systems Simulation (GloMosim) simulator will be used [27, 28]. Figure 10 and figure 11 show the GloMoSim simulator. AODV as an on- demand routing protocol and WRP as a table-driven routing protocol will be selected in the comparison. Table 2 shows summary of the simulation environments. The following is the explanation of the simulation environments.

Number of 10, 30, 50, and 75 nodes (different network size) at $200 * 300$ meter terrain. 
$>$ The routers are distributed uniformly and randomly within a terrain. All the routers are stationary and there is no network partitioning throughout the entire simulation.

Figure (10): Visualization Tool

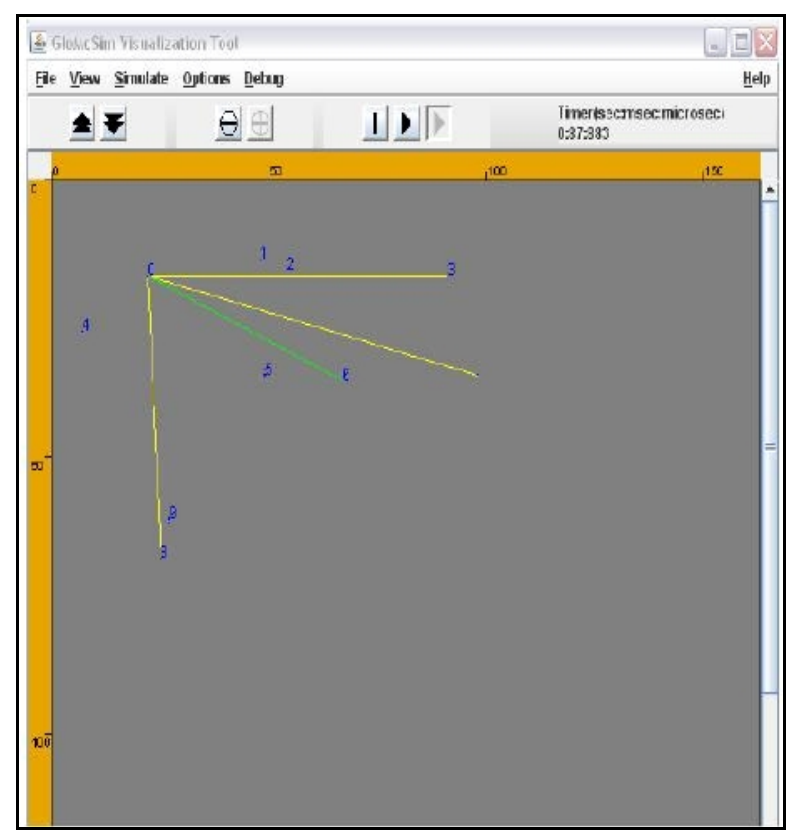

Figure (11): Running Simulation in Visualization Tool

\begin{tabular}{|c|c|}
\hline \multicolumn{2}{|c|}{ Run Simulation in Real Time } \\
\hline Executable: & glomosim config.in \\
\hline$\checkmark$ Show Radio Layer & $\nabla$ Show Network Layer \\
\hline OK & Cancel \\
\hline
\end{tabular}

\subsection{Simulation Metrics:}

To evaluate the performance of routing protocols, both qualitative and quantitative metrics are needed. Most of the routing protocols ensure the qualitative metrics. Therefore, three different quantitative metrics are used to compare the performance.

\section{1) Packet Delivery Ratio (PDR)}

The ratio of the number of data packets received by the receivers verses the number of data packets supposed to be received. This number presents the effectiveness of a protocol. 
Loss Ratio $=$

$$
\frac{\sum(\text { Bytes Received-Bytes Sent })}{\sum \text { Bytes Sent }} * 100
$$

2) Average End-to-end delay

End-to-end delay indicates how long it took for a packet to travel from the source to the receiver.

Delay $=\frac{\sum_{\mathrm{n}=1}^{\mathrm{N}} \sum_{\mathrm{p=1}}^{\mathbf{P}_{\mathrm{n}}^{\mathrm{dr}} \mathbf{D}_{\text {n.p }}}}{\sum_{\mathrm{n}=1}^{\mathrm{N}} \mathbf{P}_{\mathrm{n}}^{\mathrm{dr}}}(\mathrm{ms} /$ packet $)$

\section{3) Throughput}

The throughput is defined as the total amount of data a receiver actually receives from the sender divided by the time between receiving the first packet and last packet.

Average Network Throughput $($ Mbps $)=$

2 Nodes Recieved Bits

Number os Servers * Time Taken to recieve all bits

Table (2): Summary of Simulation Environment.

\begin{tabular}{|l|l|}
\hline Parameter & Value \\
\hline Simulator & GloMoSim \\
\hline Studied protocols & WRP, AODV, SRRP \\
\hline Simulation time & 3 M (Minutes) \\
\hline Mobility & No mobility, Mobility Existence \\
\hline Terrain dimensions & $200 \mathrm{M}, 300 \mathrm{M}$ (M:Meter) \\
\hline Number of nodes & $10,30,50,75$ \\
\hline Node placement & Random , uniform \\
\hline Propagation limit & $-111.0 \mathrm{dBm}$ \\
\hline Temperature & $290.0 \mathrm{k}$ \\
\hline Radio type & RADIO-ACCNOISE \\
\hline Radio frequency & $2.4 \mathrm{e} 9$ hertz \\
\hline Radio bandwidth & 2 Mega bit per second (Mb/s) \\
\hline RADIO-RX-TYPE & SNR-BOUNDED \\
\hline
\end{tabular}




\begin{tabular}{|l|l|}
\hline RADIO-TX-POWER & $15.0 \mathrm{dBm}$ \\
\hline RADIO-ANTENNA-GAIN & $0.0 \mathrm{~dB}$ \\
\hline
\end{tabular}

\subsection{Simulation Environment:}

This section consisted of two part, in part one the performance of the proposed routing protocol was evaluated and compared with that of the two MANET routing protocols, AODV (as on-demand protocol) and WRP(as a table driven protocol). Figures 12- 13 show this comparison. In part two, the proposed protocol will be tested over different network parameter. Figures 14- 17 show the simulation results.

\section{Part 1: The Comparison Results.}

a) The Simulation Results with no Mobility.

Figure 12 (a), shows the throughput of each protocol versus the number of nodes. It is clear from the figure that the throughput of the proposed protocol increases as the number of nodes increases since it choice to select a stable route to transmit data, while in AODV the throughput goes to be stable as the number of nodes increase and in WRP the throughput decrease as the number of nodes increase. The percentage of improvement in throughput parameter over AODV routing protocol is $21 \%$, and over WRP is $40 \%$.

Figure 12 (b), shows the average end to end delay for each protocol versus the number of nodes. It shows that the proposed protocol has the lowest delay as number of node increase, while in WRP the delay increase as the number of nodes increases, and goes to be stable in AODV. The percentage of improvement in average end-to-end delay parameter existence over AODV routing protocol is $11 \%$, and over WRP is $25 \%$.

Figure 12 (b), represents the loss ratio of packets versus the number of nodes. It is clear from the figure that with increasing the number of nodes the loss ratio decreases in the proposed protocol and goes to be stable in AODV and increases in WRP. The percentage of improvement in losing ratio parameter existence over AODV routing protocol is $23 \%$, and over WRP is $27 \%$.

b) The Simulation Results with Mobility Existence.

Figure 13(a), shows the effectiveness of the mobility in the throughput for each protocol showing that increasing number of nodes decreases the throughput for the three protocols. The proposed protocol achieves a significant improvement in throughput with increasing number of nodes. The percentage of improvement in throughput parameter existence over AODV routing protocol is $15 \%$, and over WRP is $14 \%$.

Figure13 (b), shows the effect of the mobility in the average end-to-end delay in the proposed protocol, AODV, and in WRP. Show that the proposed protocol has the 
lowest delay comparing with MANET routing protocol. The percentage of improvement in average end-to-end delay parameter over AODV routing protocol is 9\%, and over WRP is $14 \%$.

Figure 13 (c), shows the effect of the mobility in the loss ratio for each protocol (the proposed protocol, AODV, and in WRP). Show that the proposed protocol achieves the lowest packet loss ratio as number of nodes increases. The percentage of improvement in losing ratio parameter AODV routing protocol is $25 \%$, and over WRP is $24 \%$.

\section{Part 2: Testing the Proposed Protocol at different Network Parameter}

In this part, the proposed protocol will be tested at different network size in terms of the following metrics:

1) Different number of hops.

2) Mobility existence.

3) Different nodes placement.

\section{1) Different number of hops}

Figure 14 (a) show the throughput of the proposed protocol at different number of hops with different number of nodes. It shows that the throughput increases as the number of nodes increases.

Figure 14(b) represents the loss ratio of packets in the proposed protocol versus different number of hops. It is clear that as the number of hops increases the loss ratio decreases.

Figure 14(c) show average end-to-end delay in the proposed protocol versus different number of hops. It shows that the average end-to-end delay decreases as the number of hops increases. We can conclude from the last three figures that as the number of hops increases, the throughput increase, the loss ratio decreases, and the average end-to-end delay decreases.

2) Mobility Existences

Figure 15 (a) represent the throughput of the proposed protocol with and without mobility. It shows that the mobility of the nodes decreases the throughput compared with nodes with no mobility.

Figure 15 (b) shows the loss ratio of packets in the proposed protocol in both mobility and none mobility nodes. It is clear that the loss ratio decreases in the mobility nodes as there is always an alternative route.

Figure 15 (c) represents average end-to-end delay in the proposed protocol in both mobility and none mobility nodes. It shows that as the number of nodes increases the delay increases in the mobility nodes because of nodes movements.

3) Different nodes placement.

Figure 16 (a) represents the throughput of the proposed protocol with two kinds of 
nodes placement, random nodes and uniform nodes. It shows that random nodes achieve highest throughput than uniform nodes, and still increases as the number of nodes increases.

Figure 16 (b) shows the loss ratio in random nodes and uniform nodes for the proposed protocol. It is clear that as number of nodes increases the loss ratio increases in the uniform nodes in comparing with random node

Figure 16 (c) shows average end-to-end delay for both uniform and random nodes in different nodes number. It shows that as number of nodes increases the delay increases in the uniform nodes in comparing with random nodes. The conclusion of those figures is that in the proposed protocol using random nodes placement is better than uniform nodes placement.

Clearly the proposed routing protocol achieves its efficiency in different network size with different network conditions; nodes placement, number of hops, and mobility; using performance metrics (throughput, average end-to-end delay, and packet delivery loosing ratio). As a conclusion the proposed routing protocol achieve its tended performance in WMNs comparing with MANET routing protocol and in real life conditions.

Figure 12: Performance Comparison of SRRP, AODV, AND WRP.

a) Throughput of the SRRP, AODV, and WRP

b) Average End-to-End Delay for each protocol.

c) Loss Ratio of Received Packets (\%).

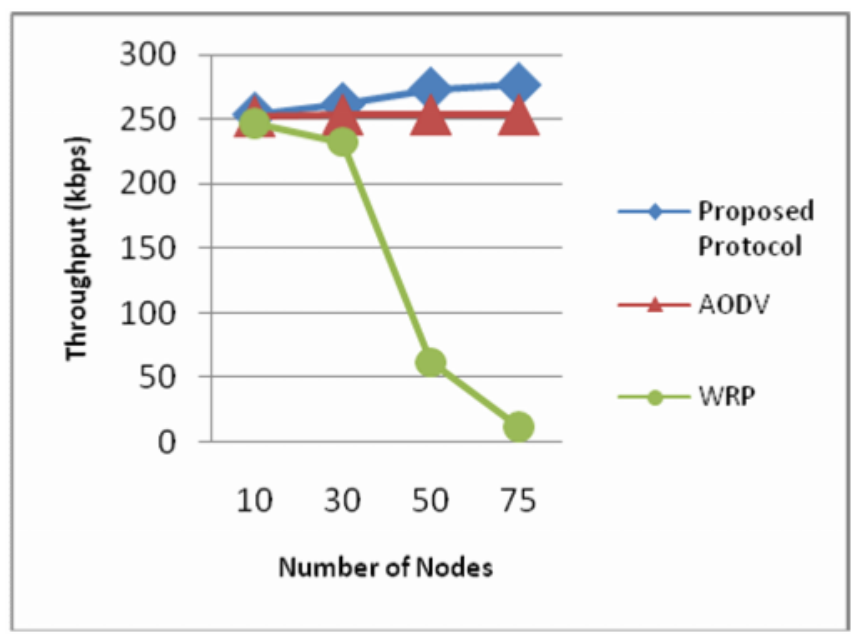

(a) 


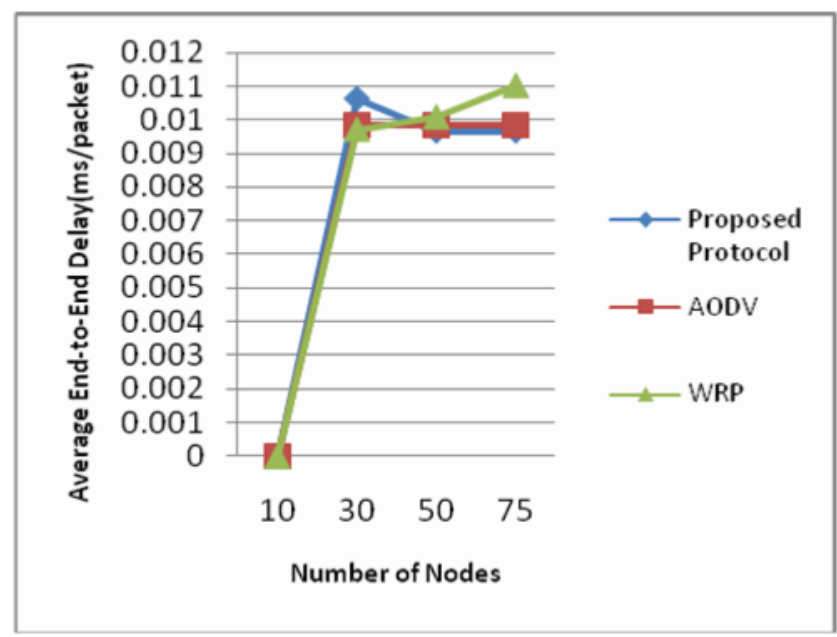

(b)

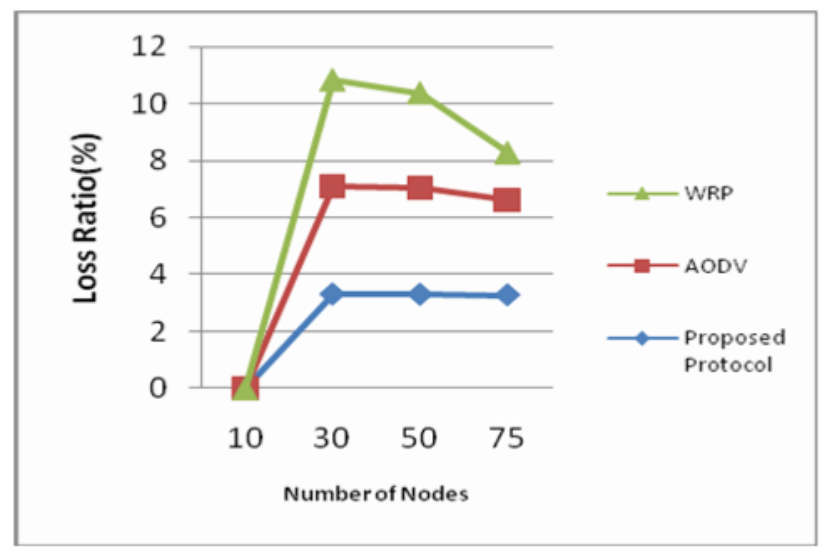

(c)

Figure 13: Performance Comparison of SRRP, AODV, and WRP with mobility existence.

a) Throughput of the SRRP, AODV, and WRP

b) Average End-to-End Delay for each protocol.

c) Loss Ratio of Received Packets (\%).

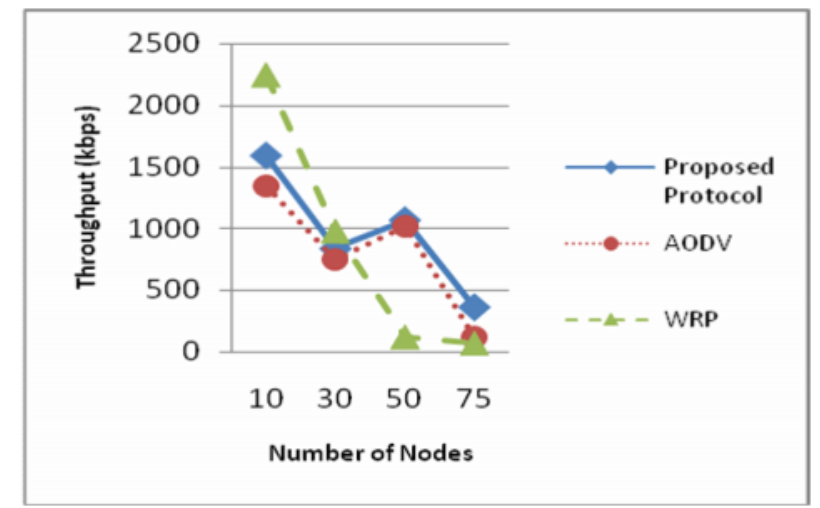

(a) 


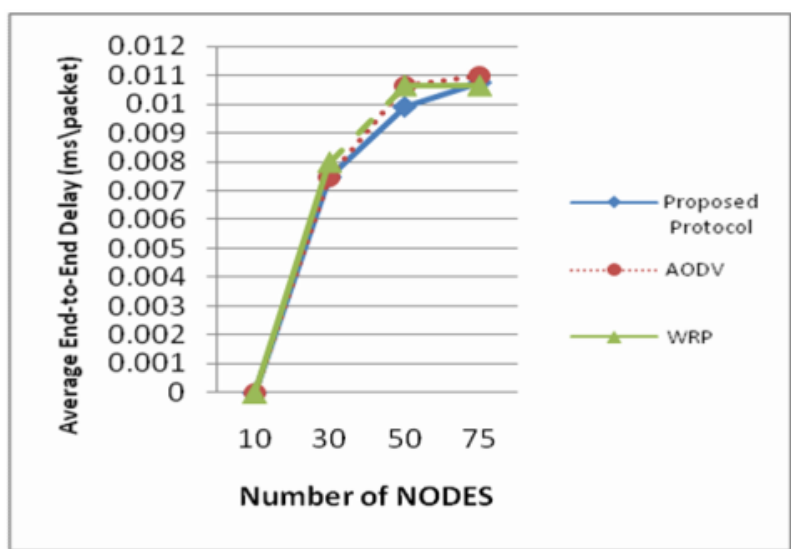

(b)

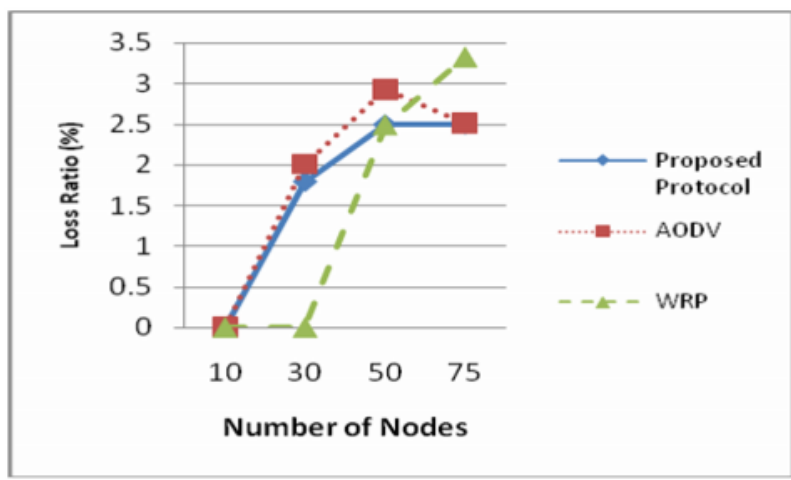

(c)

Figure 14: performance Evaluation of the Proposed Protocol against different number of hops.

a) Throughput of the SRRP in different hop number

b) Loss Ratio of Received Packets (\%).

c) Average End-to-End Delay for each protocol.

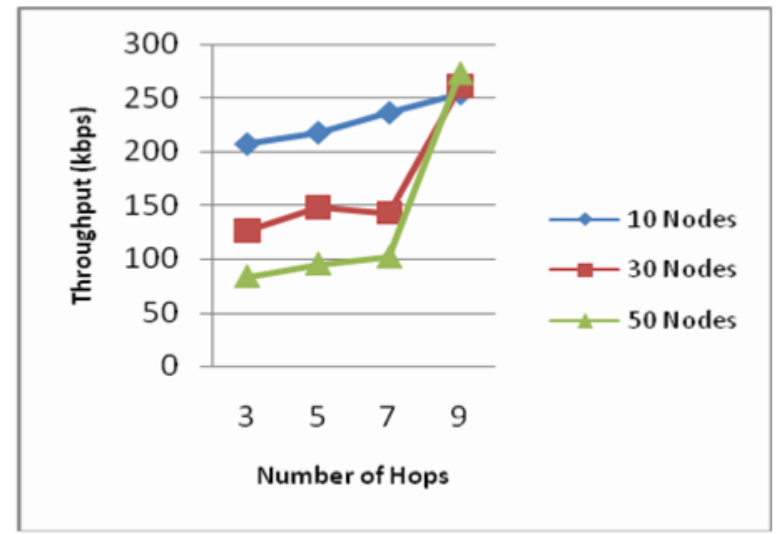

(a) 


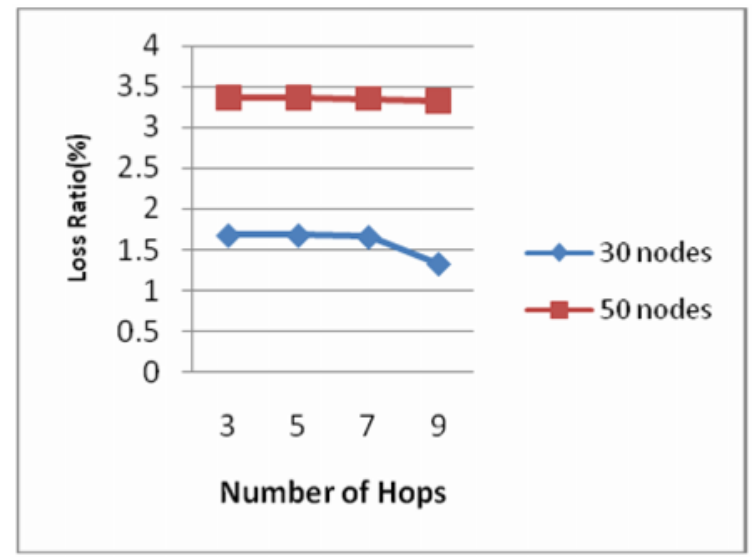

(b)

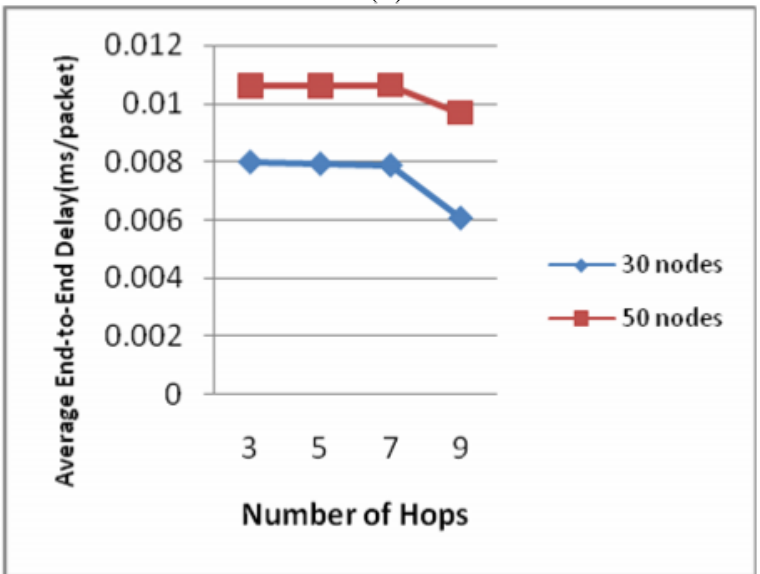

(c)

Figure 15: performance Evaluation of the Proposed Protocol against mobility existence.

a) Throughput of the SRRP in Mobility existence

b) Loss Ratio of Received Packets (\%).

c) Average End-to-End Delay for each protocol.

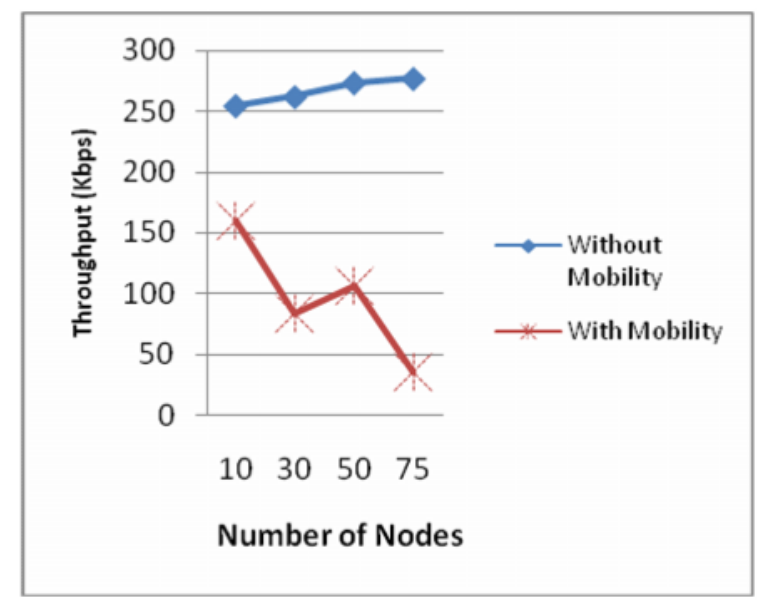

(a) 


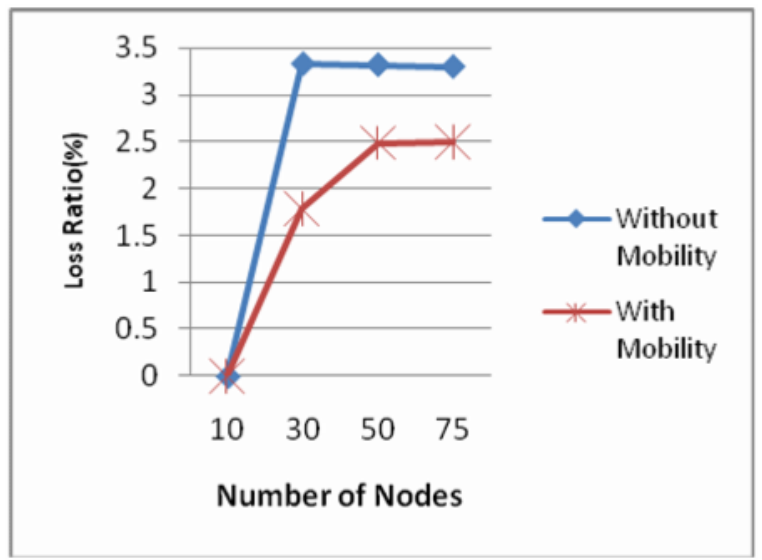

(b)

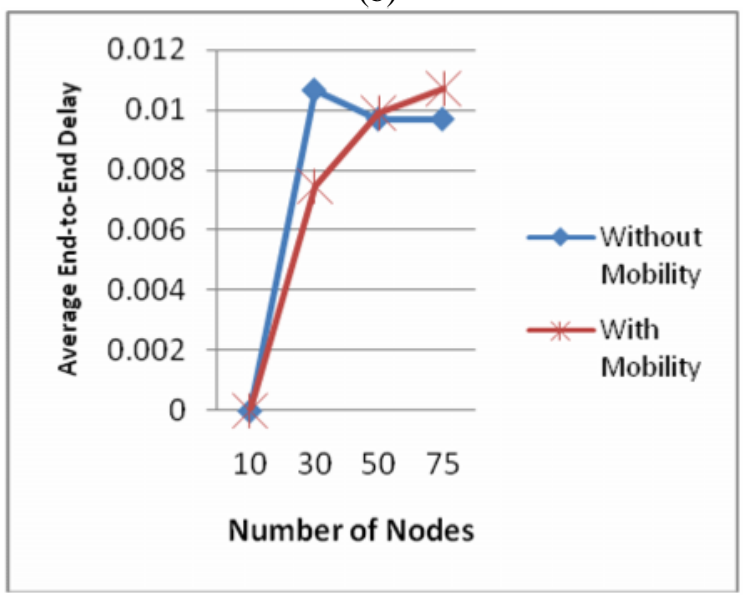

(c)

Figure 16: performance Evaluation of the Proposed Protocol against different nodes placement.

a) Throughput different nodes placement

b) Loss Ratio of Received Packets (\%).

c) Average End-to-End Delay for each protocol

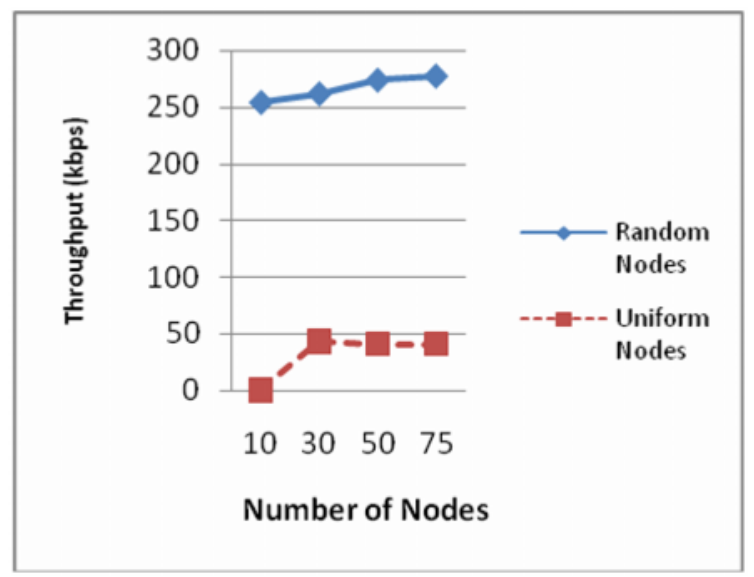

(a) 


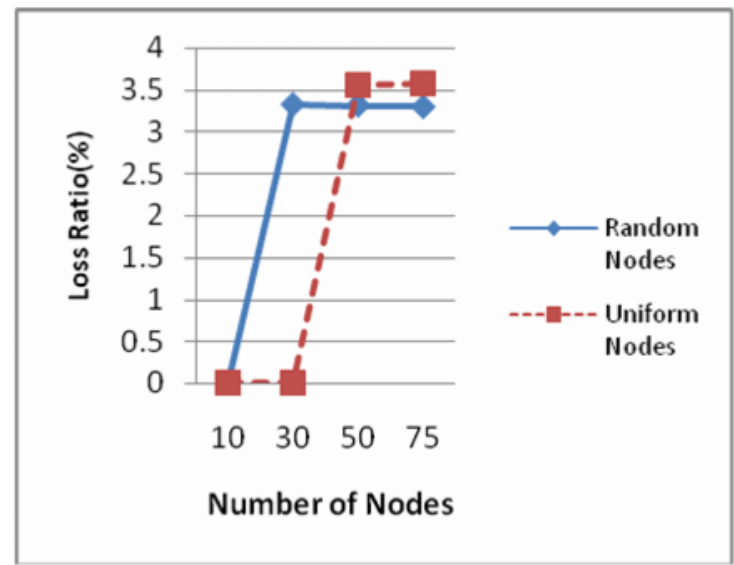

(b)

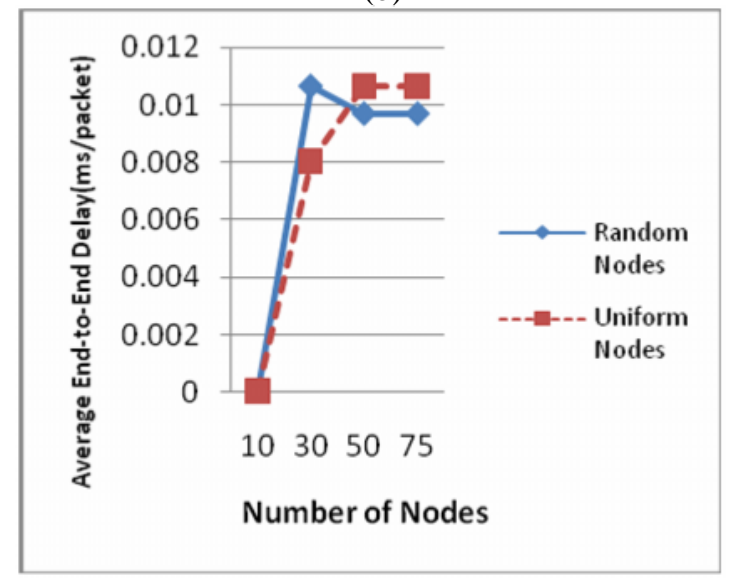

(c)

\section{Conclusion and Future Work:}

Wireless mesh networks have the potential to deliver Internet broadband access, wireless local area network coverage and network connectivity for stationary or mobile hosts at low costs both for network operators and customers. The core technology involves a network of wireless routers relaying each others' packets in a multihop fashion.

Although WMNs can be built up based on existing technologies, field trials and experiments with existing WMNs prove that the performance of WMNs is still far below expectations. As explained throughout this thesis, there still remain many research problems. Among them, the most important and urgent ones are the scalability and the mobility. Scalability based on existing MAC, routing, and transport protocols, network performance is not scalable with either the number of nodes or the number of hops in the network. This problem can be alleviated by increasing the network capacity through using multiple channels/radios per node or developing wireless radios with higher transmission speed. However, these approaches do not truly enhance the scalability of WMNs, because resource utilization is not actually improved. Therefore, 
in order to achieve scalability, it is essential to develop new MAC, routing, and transport protocols for WMNs.

Moreover, self-organization and self-configuration capability is a desired feature in WMNs. It requires protocols in WMNs to be distributive and collaborative. However, current WMNs can only partially realize this objective. Furthermore, current WMNs still have very limited capabilities of integrating heterogeneous wireless networks, due to the difficulty in building multiple wireless interfaces and the corresponding gateway/bridge functions in the same mesh router. In spite of these open research problems, we believe that WMNs will be one of the most promising technologies for next-generation wireless networking.

The proposed routing protocol SRRP based on source routing showing that by preferring stable routes, the backbone of stationary nodes presented in WMNs can be effectively used to improve the performance of routing protocol. SRRP prove its efficiency comparing with MANET routing protocols using different performance metrics. SRRP provide better throughput stability in large network with lower delay and loosing ratio in comparing with AODV and WRP as kinds of MANET routing protocols. With mobility existence, the proposed protocol achieves a significant improvement in large network comparing with MANET routing protocols.

The proposed protocol tested in different network size using different network conditions and proves to be close from achieving some of WMN expectations.

Clearly the proposed routing protocol achieves its efficiency in different network size with different network conditions; nodes placement, number of hops, and mobility; using performance metrics (throughput, average end-to-end delay, and packet delivery loosing ratio). As a conclusion the proposed routing protocol achieve its tended performance in WMNs comparing with MANET routing protocol and in real life conditions. From the comparison simulation results, the proposed routing protocol proves to achieve a significant improvement comparing with MANET routing protocols. The percentage of improvement in throughput parameter in no mobility existence over AODV routing protocol is $21 \%$, and over WRP is $40 \%$. The percentage of improvement in average end-to-end delay parameter in no mobility existence over AODV routing protocol is $11 \%$, and over WRP is $25 \%$. The percentage of improvement in losing ratio parameter in no mobility existence over AODV routing protocol is $23 \%$, and over WRP is $27 \%$. The percentage of improvement in throughput parameter in mobility existence over AODV routing protocol is $15 \%$, and over WRP is $14 \%$. The percentage of improvement in average end-to-end delay parameter in mobility existence over AODV routing protocol is $9 \%$, and over WRP is $14 \%$. The percentage of improvement in losing ratio parameter in mobility existence over AODV routing protocol is $25 \%$, and over WRP is $24 \%$. 


\subsection{Future Work:}

The following issues are to be covered in future research are presented:

1) Investigated the performance in WMNs under different performance metrics as well as different network size to understand the extent to which they influence the routing protocol effectiveness.

2) Expand the proposed routing protocol by using past knowledge to estimate the future number of request for each source-destination pair.

3) Study the different mechanisms to overcome the lower throughput in mobility nodes, and enhance the protocol efficiency in large network with mobility nodes.

\section{References:}

[1] M. L. Sichitiu, "Wireless mesh networks: Opportunities and challenge," in Proc. of the Wireless World Congress, (Palo Alto, Ca), May 2005.

[2] R. Bruno, M. Conti, and E. Gregori, "Mesh networks: Commodity multihop ad hoc networks ," IEEE Communications Magazine, vol. 43, pp. 123-131, Mar. 2005.

[3] I.F. Akyildiz, X. Wang, and W. Wang, "Wireless Mesh Networks: A Survey," Computer Networks Journal (Elsevier), March 2005.

[4] D. Beyer, "Fundamental characteristics and benefits of wireless routing ("mesh") networks," in Proc. of the International Technical Symposium of the Wireless Communications Association, (San Jose, CA), Jan. 2002.

[5] J. Jun and M. Sichitiu, "The nominal capacity of wireless mesh networks," IEEE Wireless Communication Mag., vol. 10, no. 5, pp. 8-14, Oct. 2003.

[6] M. J. Lee, J. Zheng, Y.-B. Ko, and D. M. Shrestha, "Emerging standards for wireless mesh technology," IEEE Wireless Communication Mag., vol. 13, no. 2, pp. 56-63, Apr. 2006.

[7] R. Pabst et al., "Relay-based deployment concepts for wireless and mobile broadband radio," IEEE Communication Mag., vol. 42, no. 9, pp. 80-89, Sept. 2004 
[8] A.Beyer, "Fundamental characteristics and benefits of wireless routing ("mesh") networks," in Proc. of the International Technical Symposium of the Wireless Communications Association, (San Jose,CA), Jan. 2002.

[9] L. Qiu et al., "Troubleshooting multihop wireless networks," Microsoft Research Tech. Report, MSR-TR-2004-11, Nov. 2004.

[10] B. Lewis, "Mesh networks in fixed broadband wireless access," IEEE C80216-03.10r1, July 2003.

[11] M.Richardson,"Wireless Mesh Networking ," Cisco Systems, http://www.cisco.com, 2006.

[12] V. C. Gungor, E. Natalizio, P. Pace, and S. Avallone," Challenges and Issues in Designing Architectures and Protocols forWireless Mesh Networks," www.springer.com, 2008.

[13] E.Hossain and K.Leung," Wireless Mesh Networks Architectures and Protocols," www.springer.com, 2008.

[14] GEDOC Mobile Ad Hoc Networks Study Group, "What Ad Hoc Networks are," http://www.siam.dcc.ufmg.br/gedoc/.

[15] E. Royer and C. Toh, "A review of current routing protocols for ad-hoc mobile wireless networks," IEEE Personal Communications, Apr. 1999.

[16] J. Raju and J. Garcia-Luna-Aceves, "A comparison of on-demand and tabledriven routing for ad-hoc wireless networks," in Proc. Of IEEE ICC, June 2000 .

[17] H. Xiaoyan, X. Kaixin, and M. Gerla, "Scalable routing protocols for mobile ad hoc networks,” IEEE Network, vol. 16, July-Aug 2002.

[18] C. Bhagwat, "Highly dynamic destination-sequenced distance vector routing (DSDV) for mobile computers," in Proc. of ACM SIGCOMM, pp. 234-244, Sept. 1994.

[19] C. Perkins, "Ad-hoc on-demand distance vector routing," in Proc. of MILCOM, Nov. 1997.

C. Perkins, E. Belding-Royer, and S. Das, "Ad hoc on-demand distance vector 
(AODV) routing." RFC 3561, July 2003.

[20] D. B. Johnson and D. A. Maltz, "Dynamic source routing in ad hoc wireless networks," in Mobile Computing (Imielinski and Korth,eds.), vol. 353, Kluwer Academic Publishers, 1996.

[21] Y. B. Ko and N. H. Vaidya, "Location aided routing (LAR) in mobile ad hoc networks," Wireless Networks, vol. 6, pp. 307-321, Sept. 2000.

[22] Z. J. Haas and M. R. Pearlman, "The zone routing protocol (ZRP) for ad hoc networks," internet-draft, IETF MANET Working Group, November 1997. Expiration: May, 1998.

[23] Y. Yuan, H. Yang, S. H. Y. Wong, S. Lu, and W. Arbaugh, "ROMER: resilient opportunistic mesh routing for wireless mesh networks," in Proc. of the First IEEE Workshop on Wireless Mesh Networks (WIMESH'05), (Santa Clara, CA), Sept. 2005.

[24] K. Ramachandran, M. M. Buddhikot, G. Chandranmenon, S. Miller, K. Almeroth, and E. Belding-Royer, "On the design and implementation of infrastructure mesh networks," in First IEEE Workshop on Wireless Mesh Networks (WIMESH'05), Sept. 2005.

[25] B. Cheng, M. Yuksel, and S. Kalyanaraman," Orthogonal Rendezvous Routing Protocol for Wireless Mesh Networks," 1-4244-0593-9/06/\$20.00 (C)2006 IEEE.

[27] GloMoSim: Global Mobile Information Systems Simulation Library. http://pcl.cs.ucla.edu/projects/glomosim/.

[28] Mario Gerla Lokesh Bajaj, Mineo Takai, Rajat Ahuja, and Rajive Bagrodia. GloMoSim: A Scalable Network Simulation Environment. Technical Report 990027, University of California, 13, 1999. 